\title{
COMBINAÇÃO DOS PROCESSOS ELETROCOAGULAÇÃO E FOTOCATÁLISE HETEROGÊNEA NO TRATAMENTO DE UM EFLUENTE TÊXTIL CLORADO
}

\author{
Soraya Moreno Palácio ${ }^{1}$ \\ Márcia Regina Fagundes-Klen² \\ Cláudio Celestino Oliveira ${ }^{3}$ \\ Juliana Carla Garcia ${ }^{4}$ \\ Diego Ricieri Manenti ${ }^{5}$
}

\begin{abstract}
Resumo: Este estudo investiga a degradação de um efluente têxtil clorado associando eletrocoagulação (EC), usando eletrodos de ferro, com fotocatálise heterogênea $\left(\mathrm{UV} / \mathrm{TiO}_{2} / \mathrm{H}_{2} \mathrm{O}_{2}\right)(\mathrm{FH})$, o tratamento sequencial foi chamado EC-FH. Os parâmetros operacionais dos processos foram otimizados usando experimentos fatoriais, com base no COT. O efluente foi tratado em condições ideais de cada caso, as alíquotas foram retiradas em intervalos pré-definidos de tempo para análise. A degradação foi estudada com base na descoloração, redução do COT e nitrogênio orgânico, formação de íons nitrato, amônio e sulfato. Cloro livre, cloro total e cloraminas foram determinados. A espécie Artemia salina foi utilizada para avaliar a toxicidade. A eficiência foi maior quando os processos EC e FH foram executados sequencialmente. EC removeu a maior parte do cloro total no tratamento sequencial, reduzindo a formação de cloraminas durante a FH. Mono e dicloraminas formadas no processo EC-FH foram destruídas após 180 min de reação. A toxicidade foi menor na EC-FH.
\end{abstract}

Palavras-chave: efluente têxtil; cloraminas; toxicidade; Artemia salina.

Abstract: This study investigates the degradation of a chlorinated textile effluent associating electrocoagulation (EC) using iron electrodes with heterogeneous photocatalysis $\left(\mathrm{UV} / \mathrm{TiO}_{2} / \mathrm{H}_{2} \mathrm{O}_{2}\right)$ (HP), sequential treatment was called EC-HP. The operational parameters of the process were optimized using factorial experiments based on the TOC. The effluent was treated in ideal conditions of each case, aliquots were withdrawn at predetermined time intervals for analysis. The degradation was studied based on the discoloration, removal of TOC and organic nitrogen, ions sulfate, nitrate and ammonium formation. Free chlorine, total chlorine and chloramines were determined. Artemia salina was used to assess toxicity. The pollutant removal efficiency was higher when the EC and HP processes were executed sequentially. EC removed most of the total chlorine in sequential treatment, reducing the formation of chloramines. Mono and dichloramines formed in the process EC-HP were destroyed after $180 \mathrm{~min}$ of reaction. The toxicity was lower in EC-HP.

Keywords: textile wastewater; chloramines; toxicity; Artemia salina.

\footnotetext{
1 Programa de Pós-Graduação em Engenharia Química, Universidade Estadual do Oeste do Paraná, soraya_palacio@yahoo.com.br

2 Programa de Pós-Graduação em Engenharia Química, Universidade Estadual do Oeste do Paraná, fagunde.klen@gmail.com

3 Programa de Pós-Graduação em Química, Universidade Estadual de Maringá, ccoliveira@uem.br

4 Programa de Pós-Graduação em Química, Universidade Estadual de Maringá, jucgarcia@ibest.com.br

5 Curso de Engenharia Ambiental, Universidade Federal da Fronteira Sul, diegomanenti@ hotmail.com.br
} 


\section{INTRODUÇÃO}

Atualmente existe uma grande preocupação com a preservação da qualidade da água, uma vez que o volume considerado como água potável é baixo em comparação com os totais disponíveis em todo o mundo. $O$ crescimento da população, as atividades agrícolas e industriais levam a um aumento do uso da água, que é devolvida ao ambiente, muitas vezes sem tratamento adequado, comprometendo a sua qualidade. Entre as indústrias poluentes, a têxtil tem um grande impacto ambiental devido, principalmente, a duas razões: a produção de grandes volumes de águas residuais por causa da grande quantidade de água consumida durante os processos, (Kritikos et al., 2007) e o baixo nível de fixação de matérias-primas, tais como amidos, corantes, detergentes e outros, que produzem efluentes com elevados níveis de material orgânico e forte coloração (Souza et. al., 2004).

Corantes contendo grupos azo são amplamente utilizados na indústria têxtil e devido ao potencial carcinogênico destes compostos e toxicidade dos seus intermediários de degradação, se faz necessário o tratamento dos efluentes gerados (Scott, 1995).

Muitos processos são usados para o tratamento de águas residuais contendo corantes incluindo, adsorção (Turabik, 2008; Fiorentin et al., 2010), filtração (Denyer et al., 2007), flotação (Shakir et al., 2010), coagulação (Shi et al., 2007) e métodos biológicos (Barragán et al., 2007; El-Sheekh et al., 2009).

A eletrocoagulação (EC) é um método promissor para o tratamento de efluentes têxteis, com alta eficiência de descoloração (Palácio et al., 2009), e menor produção de lodo (Wu et al., 2008). O processo de eletrocoagulação foi relatado por vários outros autores no tratamento de águas residuais contendo corantes (Kim et al., 2002; Mollah et al., 2004; Golder et al., 2005; Daneshvar et al., 2006).

$\mathrm{Na}$ EC aplica-se uma corrente elétrica para produzir íons metálicos em solução. Os íons metálicos podem reagir com os íons $\mathrm{OH}^{-}$formados no cátodo durante a evolução de gás hidrogênio produzindo hidróxidos insolúveis que irão adsorver os poluentes. As reações que ocorrem durante a EC podem ser resumidas pelas reações 1 e 2 (Kurbus et al., 2003).

$$
\begin{aligned}
& \text { Ânodo: } \mathrm{M} \rightarrow \mathrm{M}^{\mathrm{n}+}+\mathrm{ne}^{-} \\
& \text {Cátodo: } \mathrm{nH}_{2} \mathrm{O}+\mathrm{ne}^{-} \rightarrow \mathrm{n} / 2 \mathrm{H}_{2}+\mathrm{nOH}^{-}
\end{aligned}
$$

em que $\mathrm{M}$ é o metal que constitui o ânodo e n é o número de elétrons envolvidos na reação.

Tratamentos destrutivos tais como os processos oxidativos avançados (POAs) podem ser aplicados ao tratamento de efluentes têxteis reduzindo espécies químicas em fragmentos menores e, eventualmente, atingindo completa mineralização do poluente (Garcia et al., 2007). A degradação do poluente ocorre através da ação de espécies altamente oxidantes tais como os radicais hidroxila gerados no meio reacional. Os POAs mais comumente usados são sistemas $\mathrm{UV} / \mathrm{H}_{2} \mathrm{O}_{2}, \mathrm{UV} / \mathrm{O}_{3} \mathrm{e}$ $\mathrm{O}_{3}$ (Azbar et al., 2004.), reações de Fenton e foto-Fenton (Papic et al., 2009) e fotocatálise heterogênea utilizando $\mathrm{TiO}_{2}$ (Garcia et al., 2009) e ZnO (Chakrabarti e Dutta, 2004; Qamar et al., 2005; Rodrigues et al., 2008).

Desta forma, o principal objetivo deste estudo foi investigar a degradação de um efluente têxtil clorado associando eletrocoagulação (eletrodos de ferro) com fotocatálise heterogênea $\left(\mathrm{UV} / \mathrm{TiO}_{2} / \mathrm{H}_{2} \mathrm{O}_{2}\right)$.

\section{MATERIAIS E MÉTODOS}

\subsection{AMOSTRAGEM}

Amostras de efluente foram coletadas em vários dias consecutivos em uma lavanderia industrial. Uma mistura de todas as amostras foi feita a fim de se obter um efluente têxtil não tratado (ET) homogêneo e representativo dos processos realizados pela indústria. 
O ET coletado continha corantes do tipo direto, contendo grupamentos azo, além de cloreto de sódio, hidróxido de sódio, hipoclorito de sódio e surfactantes, como componentes principais. Antes dos tratamentos, todas as amostras do ET, foram preservadas de acordo com as metodologias descritas no Standad Methods (APHA, 2005). Alíquotas do ET foram utilizadas para caracterização.

$\mathrm{O} \mathrm{TiO}_{2}$ utilizado foi P-25 $(80 \%$ anatase e $20 \%$ rutilo) com uma superfície específica de $50 \mathrm{~m}^{2} \mathrm{~g}^{-1}$ que foi fornecido pela Evonik-Degussa-Brasil e utilizado sem purificação prévia. $\mathrm{O}_{2} \mathrm{H}_{2} \mathrm{O}_{2}(30 \%$, grau analítico) utilizado foi da marca Synth. Os outros reagentes utilizados tinham qualidade analítica.

\subsection{ELETROCAGULAÇÃO (EC)}

Foi utilizado um reator em escala laboratorial de vidro cilíndrico com capacidade de uma 1,5 L possuindo 11 $\mathrm{cm}$ de diâmetro e $15 \mathrm{~cm}$ de altura. Foram usadas como eletrodos oito placas de ferro com 5,0 cm de largura de, 11,0 de altura e 0,15 de espessura. $\mathrm{Na}$ parte superior do reator de EC, as placas de eletrodos de ferro foram firmemente montadas numa posição vertical e dispostas paralelamente umas às outras, com um intervalo de $0,5 \mathrm{~cm}$ entre as placas do ânodo e do cátodo, utilizando um suporte horizontal não condutor para evitar curtos-circuitos (Fig. 1). Os eletrodos foram mergulhados no reator contendo um volume de trabalho de $1,0 \mathrm{~L}$ de efluente têxtil. A área de superfície ativa do eletrodo foi de $0,035 \mathrm{~m}^{2}$. Os eletrodos foram operados em modo monopolar e foram conectados aos terminais de energia com alimentação direta de corrente (Instrutemp DC Power Supply, FA 1030), que continham um amperímetro interno digital e voltímetro, que forneceu as correntes e tensões estabilizadas, variando de 0 a $10 \mathrm{~A}$, e entre 0 e $30 \mathrm{~V}$, respectivamente. Durante os experimentos a direção da corrente foi invertida a cada 30 minutos, a fim de reduzir a passivação dos eletrodos pela formação de camadas de óxidos nas suas superfícies (Chen, 2004).

$\mathrm{O}$ processo de tratamento $\mathrm{EC}$ foi realizado considerando as condições ótimas obtidas por meio de um planejamento fatorial $3^{3}$ completo em experimento prévio (Palácio et al., 2009), sendo os valores operacionais de 142,9 $\mathrm{m}^{-2}$ de densidade de corrente, $\mathrm{pH} 7$ e 60 min de eletrocoagulação.

Utilizando as condições de funcionamento da EC, o efluente têxtil foi eletrocoagulado e submetido posteriormente ao tratamento por fotocatálise heterogênea usando $\mathrm{UV} / \mathrm{TiO}_{2} / \mathrm{H}_{2} \mathrm{O}_{2}$.

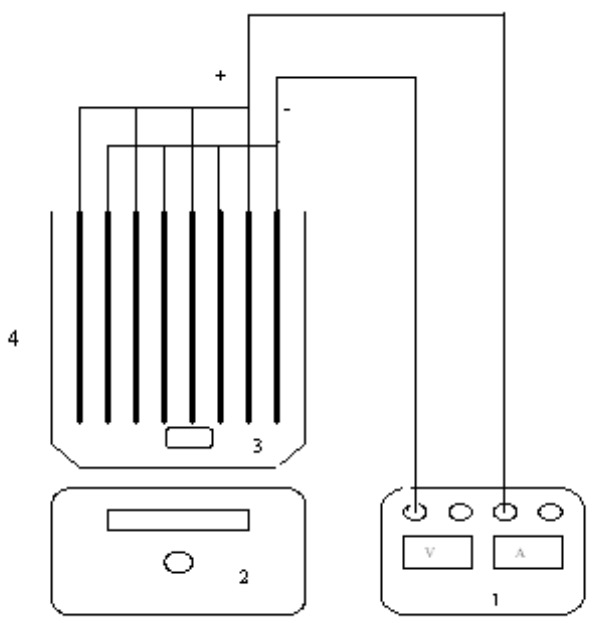

Figura 1. Diagrama esquemático do arranjo experimental: (1) Fonte estabilizada de corrente contínua (V, regulador de voltagem e $\mathrm{A}$, regulador de corrente); (2) agitador magnético; (3)

barra magnética; (4) célula de eletrocoagulação. Fonte: Palácio et al., 2013.

\subsection{PROCESSO DE ELETROCOAGULAÇÃO ASSOCIADO COM A (EC-FH).}

A irradiação foi realizada em reator aberto, cilíndrico, de vidro borosilicato e com capacidade de $1 \mathrm{~L} \mathrm{em}$ condições ambientes . Os reagentes juntamente com o efluente foram continuamente agitados magneticamente no reator fotoquímico. A superfície das soluções ou suspensões ficou a aproximadamente $30 \mathrm{~cm}$ de distância das 
fontes de radiação (três lâmpadas de mercúrio de $250 \mathrm{~W}$ alta pressão sem o bulbo). As lâmpadas foram fixadas verticalmente na parede do topo de uma caixa de madeira $(80 \times 80 \times 50 \mathrm{~cm})$. Quatro coolers, do tipo usado em computadores, foram instalados em diferentes posições nas paredes laterais do reator para minimizar o efeito de aquecimento produzido pelas lâmpadas (Fig. 2). As paredes internas da caixa foram cobertas com chapas de alumínio polido para evitar as perdas de radiação e a temperatura interna variou aproximadamente $24{ }^{\circ} \mathrm{C}$ durante $\mathrm{o}$ processo de irradiação, sendo os valores médios iniciais de $23{ }^{\circ} \mathrm{C}$ e os finais de 47 ${ }^{\circ} \mathrm{C}$.

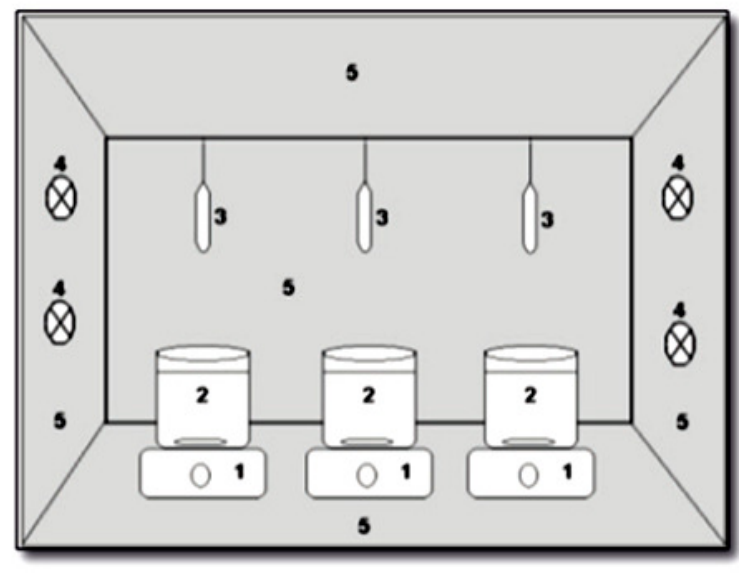

Figura 2. Sistema utilizado para as reações foto-Fenton com irradiação artificial. Sendo: 1 = agitadores magnéticos; 2 = reatores foto-Fenton; $3=$ lâmpadas de mercúrio; 4 = ventiladores; 5 = chapas de alumínio polido. Adaptado de Palácio et al, 2012.

Para otimizar os parâmetros de operação do reator (POR), foi realizado um planejamento fatorial $3^{2} \mathrm{com}$ as amostras eletrocoaguladas em triplicata. Os níveis dos POR pré-definidos para a concentração de $\mathrm{TiO}_{2}$ foram: $0,10,0,25$ e $0,50 \mathrm{~g} \mathrm{~L}^{-1}$ e $\mathrm{H}_{2} \mathrm{O}_{2}$ foram: $0,001,0,010 \mathrm{e}$ $0,100 \mathrm{~mol} \mathrm{~L}^{-1}$. Antes da irradiação, 500 $\mathrm{mL}$ de suspensões (efluente $+\mathrm{TiO}_{2}$ ) foram sonicadas em banho de ultrassom (UNIQUE modelo USC-1400) durante 15 minutos no escuro, a um $\mathrm{pH}$ de 3,0 ajustado com $\mathrm{H}_{2} \mathrm{SO}_{4}$ 0,1 mol L $\mathrm{L}^{-1} \mathrm{e}$ de $\mathrm{NaOH} 0,1 \mathrm{~mol} \mathrm{~L}^{-1}$ (pH obtidos em testes preliminares de adsorção). Após a adsorção, o $\mathrm{H}_{2} \mathrm{O}_{2}$ foi adicionado às suspensões e as amostras foram irradiadas por 6 horas. O parâmetro analisado foi a redução de carbono orgânico total (COT).

Usando os valores ótimos dos POR o efluente foi irradiado por 60, 120, 180, 240, 300 e 360 min. Nas amostras obtidas em cada tempo foram determinados os parâmetros: $\mathrm{pH}, \mathrm{COT}$, DQO, turbidez, cor, sulfato, nitrato, nitrogênio Kjeldahl total, nitrogênio orgânico, nitrogênio amoniacal, cloro livre, monocloraminas, dicloraminas, toxicidade utilizando a espécie Artemia salina.

Embora a fotocatálise heterogênea $(\mathrm{FH})$ não seja um processo adequado para o tratamento de efluentes com DQO acima de $800 \mathrm{mg} \mathrm{L}^{-1}$ (Gogate e Panditi, 2004), as águas residuais não tratadas $(\mathrm{DQO}=1636 \pm 87)$ foram diluídas com água destilada para se obter uma DQO de $800 \mathrm{mg} \mathrm{L}^{-1}$ e submetidos a este processo, a fim de avaliar a formação de cloraminas e sua relação com a toxicidade do efluente. A otimização das variáveis operacionais seguiu o mesmo planejamento fatorial descrito acima, utilizando o COT como resposta. O efluente foi irradiado por $60,120,180$, 240, 300 e 360 min nas condições ótimas dos POR. Nas amostras obtidas em cada tempo foram determinados os parâmetros: cloro livre, monocloraminas, dicloraminas e toxicidade utilizando a espécie Artemia salina.

O cálculo dos valores ótimos dos POR foi realizado utilizando o pacote estatístico Statistica (Copyright 19842000 by statsoft, Inc) no modo "experimental design".

\subsection{DETERMINAÇÕES ANALÍTICAS}

Todas as determinações analíticas foram efetuadas em triplicata. $\mathrm{O} \mathrm{pH}$ foi medido usando um medidor digital de $\mathrm{pH}$ (modelo Tecnal TEC-2). O carbono orgânico total (COT) foi medido utilizando um analisador de carbono orgânico total (Shimadzu modelo TOCL). A demanda química de oxigênio (DQO) foi determinada pelo método 
colorimétrico de refluxo fechado, como descrito no Standard Methods (APHA, 2005). Esta metodologia foi aferida em quintuplicatas analíticas usando uma solução padrão (Combicheck 20) com $750 \pm 75 \mathrm{mg}$ de $\mathrm{O}_{2} \mathrm{~L}^{-1}$. O resultado médio foi de $710 \pm 24 \mathrm{mg}$ de $\mathrm{O}_{2} \mathrm{~L}^{-1}$. A turbidez foi determinada pelo método nefelométrico (Nephelometric Turbidity Unit, NTU) utilizando um turbidímetro (Tecnal, modelo TB1000). Medida de condutividade elétrica foi realizada utilizando um condutivímetro (Tecnal, modelo TEC-4P-MP). A cor foi determinada pelo método de platinacobalto de acordo com a metodologia padrão e as concentrações de íons sulfato foram determinados pelo método turbidimétrico, ambos os métodos estão descritos no Standard Methods (APHA, 2005).

As quantidades de nitrato foram medidas utilizando o método da cinética de redução do cádmio, enquanto que um método colorimétrico baseado na reação fenato-hipoclorito foi utilizado para determinar a concentração de nitrogênio amoniacal. O nitrogênio Kjeldahl total foi determinado com base num processo de digestão ácida (APHA, 2005) e a concentração de nitrogênio orgânico, foi determinada por subtração.

As concentrações de cloro total, cloro livre, monocloraminas e dicloraminas foram medidas utilizando o método titulométrico de N, N-dietil-pfenilenodiamina (DPD) (APHA, 1998).

A concentração de $\mathrm{H}_{2} \mathrm{O}_{2}$ foi determinada utilizando o método do metavanadato de amônio (Oliveira et al, 2001). Este método baseia-se na reação do $\mathrm{H}_{2} \mathrm{O}_{2}$ com metavanadato em meio ácido, formando o cátion peroxovanádio de cor vermelho alaranjado que apresenta absorbância máxima em $450 \mathrm{~nm}$.

As absorbância em $420 \quad \mathrm{~nm}$ (método turbidimétrico), $430 \mathrm{~nm}$ (método de Platina-Cobalto), $450 \mathrm{~nm}$ (método de metavanadato), $510 \mathrm{~nm}$ (método fenantrolina) e $600 \mathrm{~nm}$ (método colorimétrico refluxo fechado), bem como para determinar as formas de nitrogênio foram obtidas utilizando um espectrofotômetro UV-Vis (Shimadzu, modelo UV-1601 PC).

Antes das análises, todas as amostras foram filtradas utilizando membranas de nylon (porosidade $25 \mathrm{~mm}$ e 0,45 $\mathrm{mm}$ de diâmetro), exceto para a determinação de COT. Nas determinações envolvendo oxidação química (Vilar et al., 2011), o excesso de $\mathrm{H}_{2} \mathrm{O}_{2}$ presente nas amostras foi removido pela adição de uma alíquota $\left(0,1 \mathrm{~g} \mathrm{~L}^{-1}\right)$ da solução de catalase $(2500 \mathrm{U}$ fígado bovino $\mathrm{mg}^{-1}$ ).

\subsection{TESTE DE TOXICIDADE COM A ESPÉCIE ARTEMIA SALINA}

O bioensaio com a espécie Artemia salina (A. salina) foi utilizado para avaliar o efeito de letalidade dos efluentes tratados e não tratado, baseado no critério de $\mathrm{LC}_{50}$. Os cistos de A. salina foram deixados para eclodir em solução nutriente descrita em Meyer (1982) por 48 horas, as larvas mais resistentes foram usadas nos testes de toxicidade. Para cada amostra de efluente não tratado e tratados (EC, FH e EC-FH), foi preparado $5 \mathrm{ml} \mathrm{de}$ uma mistura de efluentes e a solução nutriente (v/v) em um tubo de vidro de 10 $\mathrm{ml}$, nas seguintes proporções: $20 \%, 40 \%$, $60 \%, 80 \%$ e $100 \%$, em triplicata. Em cada tubo contendo as diluições, 10 larvas de A. salina foram incubadas à temperatura ambiente e regime de luz, durante $24 \mathrm{~h}$. Após este período foram contadas as larvas vivas e mortas e calculada a $\mathrm{LC}_{50}$, conforme a metodologia descrita por Hamilton et al. (1997), utilizando-se o software Trimmed Spearman-Karber Method Versão 1.5. Como teste controle negativo, 10 larvas de A. salina, foram incubadas na solução nutriente recém preparada.

\section{RESULTADOS E DISCUSSÃO}

O efluente têxtil foi usado no processo de eletrocoagulação sem qualquer diluição prévia e as suas características são apresentadas na Tabela 1. 
Tabela 1. Características físico-químicas do efluente têxtil não tratado.

\begin{tabular}{|c|c|}
\hline Parâmetros & Valor \\
\hline $\mathrm{pH}$ & 12,5 \\
\hline Turbidez (NTU) & 306 \\
\hline $\begin{array}{l}\text { Nitrogênio orgânico }\left(\mathrm{mg} \mathrm{NH}_{4}{ }^{+} \mathrm{L}^{-}\right. \\
\left.{ }^{1}\right)\end{array}$ & 4,6 \\
\hline Amônia $\left(\mathrm{mg} \mathrm{NH}_{4}^{+} \mathrm{L}^{-1}\right)$ & 1,3 \\
\hline$\left(\mathrm{C} \mathrm{L}^{-1}\right)$ & 636 \\
\hline $\mathrm{DQO}\left(\mathrm{mg} \mathrm{O}_{2} \mathrm{~L}^{-1}\right)$ & 1636 \\
\hline $\left.\mathrm{O}_{2} \mathrm{~L}^{-1}\right)$ & 328 \\
\hline $\mathrm{DQO}^{-\mathrm{DBO}_{5}}$ & 0,2 \\
\hline Sulfato $\left(\mathrm{mg} \mathrm{SO}_{4}{ }^{2-} \mathrm{L}^{-1}\right)$ & 2681 \\
\hline $\operatorname{Cor}(\mathrm{g} \mathrm{Pt} \mathrm{Co} \mathrm{L} \mathrm{L-1})$ & 5,100 \\
\hline $1\left(\mathrm{mg} \mathrm{Cl}_{2} \mathrm{~L}^{-1}\right)$ & 1905 \\
\hline Condutividade elétrica $\left(\mathrm{mS} \mathrm{cm}^{-1}\right)$ & 16,88 \\
\hline
\end{tabular}

A relação $\mathrm{DQO}^{-\mathrm{DBO}_{5}}$ (usada como índice de biodegradabilidade) foi de 0,2, causada pelo elevado valor de DQO. Esse índice baixo indica a presença de substâncias refratárias, provavelmente compostos orgânicos estáveis, os quais dificilmente podem sofrer degradação biológica (Economides et al., 1998). Valores do índice de biodegradabilidade inferiores a 0,3 indicam que o efluente não é adequado para o tratamento biológico (Karrer et al., 1997). Além disso, o valor elevado de DQO (1636 mg $\mathrm{L}^{-1}$ ) torna inadequado o tratamento deste efluente por fotocatálise usando o $\mathrm{TiO}_{2}$. Desta forma, foi proposto o tratamento preliminar pelo processo de eletrocoagulação.

Além disso, os dados da Tabela 1 mostram a presença de grandes quantidades de íons sulfato e cloro total. O cloro provém da adição de cloreto de sódio e hipoclorito de sódio durante os processos de tingimento e desbotamento.

$\mathrm{O}$ efluente pré-tratado pelo processo EC foi tratado posteriormente pelo processo $\mathrm{FH}$ constituindo o EC-FH.
As respostas obtidas para a redução do COT foram avaliadas com o auxílio do software Statistica ${ }^{\circledR}$. Os valores ótimos dos parâmetros operacionais do reator calculados pelo planejamento fatorial para a redução de COT foram $0,25 \mathrm{~g} \mathrm{~L}^{-1}$ de $\mathrm{TiO}_{2}$ e 0,03 mol L $\mathrm{L}^{-1}$ de $\mathrm{H}_{2} \mathrm{O}_{2}$, para amostras eletrocoaguladas. No processo FH utilizando o efluente têxtil diluído os valores ótimos para os POR foram $0,50 \mathrm{~g}$ $\mathrm{L}^{-1}$ de $\mathrm{TiO}_{2}$ e $0,05 \mathrm{~mol} \mathrm{~L}^{-1} \mathrm{H}_{2} \mathrm{O}_{2}$.

\subsection{DEGRADAÇÃO DO EFLUENTE TÊXTIL PELOS PROCESSOS EC E EC-FH}

A remoção máxima do COT no processo de EC foi de $43 \%$ e no EC-FH $66 \%$ (Fig. 3a) operando nas condições ótimas dos POR. Na EC há uma predominância de remoção por coagulação com a formação de lodo, porém podem ter ocorrido reações de oxidação-redução contribuindo para a remoção do COT por mineralização. $\mathrm{Na}$ EC-FH a mineralização do COT ocorreu somente após $300 \mathrm{~min}$ de irradiação, promovendo a oxidação de $23 \%$ do COT remanescente após a EC.

A remoção de nitrogênio orgânico nos processos de EC e EC-FH foi de 41 e $85 \%$, respectivamente (Fig. 3b). A formação de nitrato na EC está relacionada com a oxidação da matéria orgânica pelo processo de oxidaçãoredução que ocorre na eletrocoagulação. No início do processo EC-FH ocorre uma diminuição da concentração de nitrato devido à sua adsorção nas partículas de $\mathrm{TiO}_{2}$ (Garcia et al., 2006). O aumento deste íon a partir de 180 min sugere a mineralização do nitrogênio orgânico remanescente. 


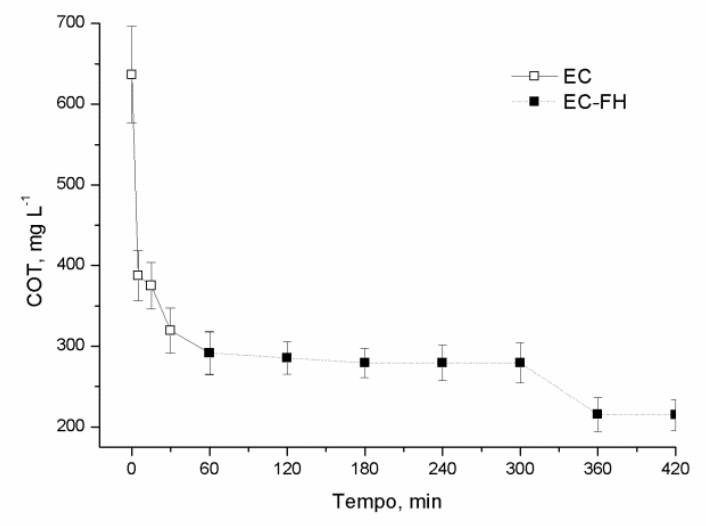

(a)

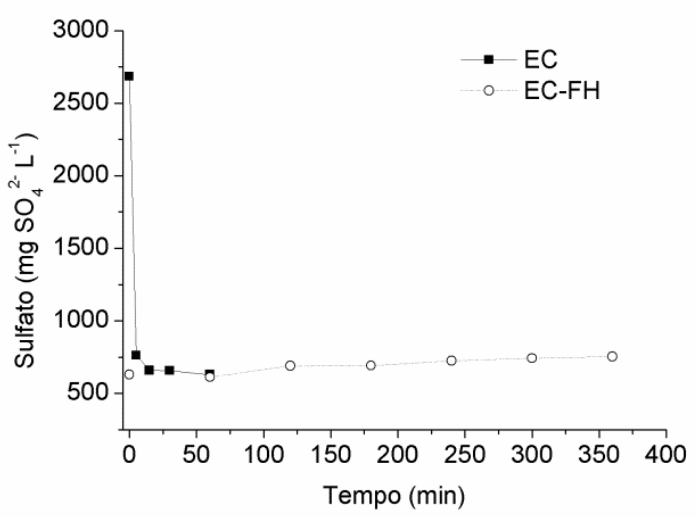

(c)

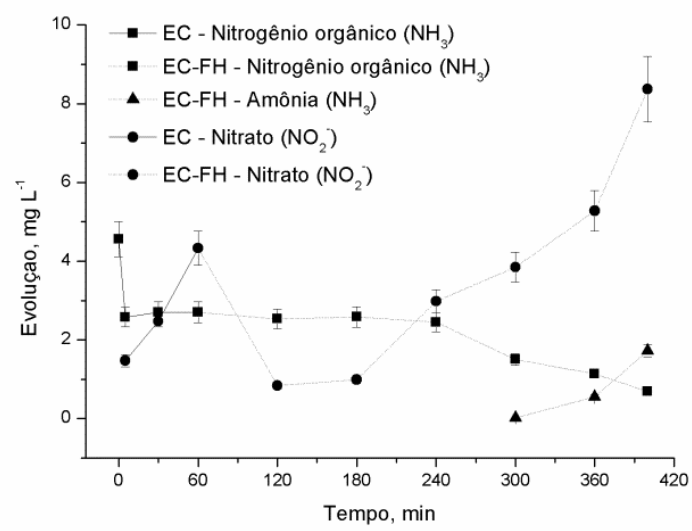

(b)

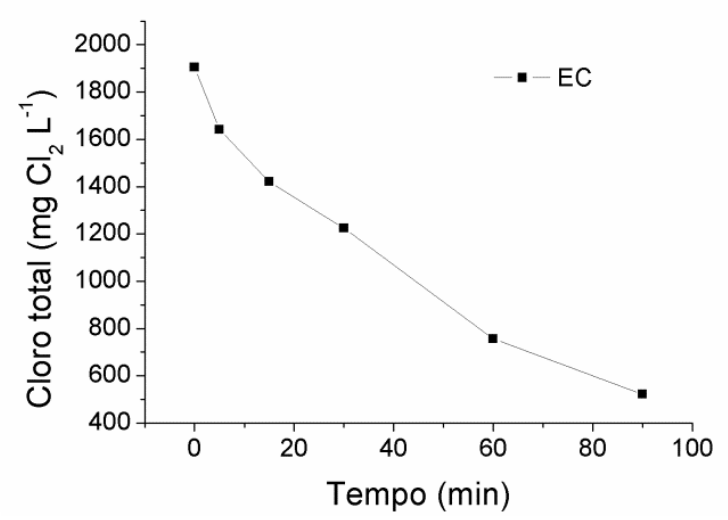

(d)

Figura 3. (a) Valores de COT (mg L $\left.{ }^{-1}\right)$ para EC e EC-FH; (b) Evolução das formas nitrogenadas: nitrogênio orgânico $\left(\mathrm{mg} \mathrm{NH}_{3} \mathrm{~L}^{-1}\right)$, nitrogênio amoniacal $\left(\mathrm{mg} \mathrm{NH}_{3} \mathrm{~L}^{-1}\right)$ e nitrato $\left(\mathrm{mg} \mathrm{NO}_{3}{ }^{-} \mathrm{L}^{-1}\right)$ para EC and EC-FH; (c) Valores de Sulfato $\left(\mathrm{mg} \mathrm{L}^{-1}\right)$ para EC e EC-FH e (d)

Concentrações de Cloro total $\left(\mathrm{mg} \mathrm{Cl}_{2} \mathrm{~L}^{-1}\right)$ para $\mathrm{EC}$, em função do tempo de reação.

A remoção de sulfato pela EC foi elevada, alcançando $71,6 \%$ nos primeiro $5 \mathrm{~min}$, reduzindo de 2684 para $761 \mathrm{mg} \mathrm{SO}_{4}{ }^{2-} \mathrm{L}^{-1}$ (Fig. 3c). No tempo de 60 min a remoção foi de $76,5 \%$. No EC-FH foi observado na primeira hora de irradiação uma diminuição de $2,7 \%$ na concentração de íons sulfato, provavelmente pela adsorção destes íons no $\mathrm{TiO}_{2}$ (Garcia et al., 2006). A formação de íons sulfato observada nos tempos de irradiação subsequentes indicam uma possível mineralização de compostos orgânicos contendo enxofre em 120 min de irradiação com um aumento de $18,81 \%$ e novamente podemos observar que a fotodegradação ocorre após a redução da carga poluidora pelo processo EC.
O efluente têxtil não tratado apresentou elevada concentração de cloro total (1905 $\left.\quad \mathrm{mg} \quad \mathrm{Cl}_{2} \quad \mathrm{~L}^{-1}\right)$. Elevadas concentrações de cloro no efluente pode proporcionar, na presença de compostos nitrogenados a formação de cloraminas. As cloraminas (mono e di) são indesejáveis por causa de sua ação desinfetante (USEPA, 1994), podendo afetar a eficiência do tratamento biológico ou causar danos aos organismos aquáticos presentes no corpo receptor. A remoção prévia do cloro previne a formação das cloraminas.

As cloraminas são formados pela reação do ácido hipocloroso e amônia, de acordo com reações 3-5 para mono, di e 
tricloramina, respectivamente (White, 1999).

$$
\begin{aligned}
& \mathrm{NH}_{3}+\mathrm{HOCl} \rightarrow \mathrm{NH}_{2} \mathrm{Cl}+\mathrm{H}_{2} \mathrm{O} \\
& \mathrm{NH}_{2} \mathrm{Cl}+\mathrm{HOCl} \rightarrow \mathrm{NHCl}_{2}+\mathrm{H}_{2} \mathrm{O} \\
& \mathrm{NHCl}_{2}+\mathrm{HOCl} \rightarrow \mathrm{NCl}_{3}+\mathrm{H}_{2} \mathrm{O}
\end{aligned}
$$

No processo EC os valores de cloro total diminuíram de 1905 para $522 \mathrm{mg} \mathrm{Cl} 2$ $\mathrm{L}^{-1}(72,3 \%)$ no tempo de 90 min e para 755 $(60,3 \%)$ no tempo de $60 \mathrm{~min}$. Acredita-se que a remoção tenha ocorrido por oxidação com formação de $\mathrm{Cl}_{2}$. No processo EC íons cloreto, com carga negativa são atraídos para o ânodo, onde perde um elétron originando o gás cloro $\left(\mathrm{Cl}_{2}\right)$ (reação 6):

$$
2 \mathrm{Cl}^{-} \rightarrow \mathrm{Cl}_{2}(\mathrm{~g})+2 \mathrm{e}^{-}
$$

Cloro livre, mono e dicloraminas não foram detectados nos efluentes tratados por EC. O cloro livre foi detectado somente no processo EC-FH nas concentrações de 3 e $2 \mathrm{mg} \mathrm{Cl}_{2} \mathrm{~L}^{-1}$ quando as amostras foram irradiadas por 60 e 120 min, respectivamente. No processo $\mathrm{FH}$, utilizando o efluente diluído, houve a formação de quantidades muito maiores de mono e dicloraminas (Tab. 2) quando comparado com o processo EC-FH.
As proporções de espécies de cloraminas formadas dependem do $\mathrm{pH}$. Os resultados mostraram que houve a formação predominante de dicloraminas, sendo que o $\mathrm{pH}$ do meio reacional é aproximadamente 3,0 para $\mathrm{FH}$ e EC-FH, concordando com Palin (1950) que afirma ser a dicloramina a espécie dominante em $\mathrm{pH}$ inferior a 5 e monocloroamina o principal componente em pH entre 6 e 8 .

Alta eficiência na remoção do cloro em compostos bifenílicos policlorados utilizando o tratamento eletroquímico foi demonstrada por Zhang e Rusling (1995) contribuindo para a diminuição da toxicidade do efluente. Polcaro et al. (1999) também obtiveram uma boa eficiência na remoção de 2-clorofenol de efluentes industriais.

Neste estudo, a remoção parcial de cloro na EC contribuiu para a redução da formação de cloraminas no tratamento posterior (Fig. 3d). Em EC-FH monocloraminas foram formadas antes de 180 minutos de irradiação, sendo máxima em $120 \mathrm{~min}\left(5,0 \mathrm{mg} \quad \mathrm{Cl}_{2} \quad \mathrm{~L}^{-1}\right)$ e as dicloraminas somente em $180 \mathrm{~min}(5,0 \mathrm{mg}$ $\mathrm{Cl}_{2} \mathrm{~L}^{-1}$ ). Com 240 min de irradiação tanto as mono, como as dicloraminas foram

\begin{tabular}{|c|c|c|c|c|c|c|}
\hline \multirow[t]{2}{*}{$\begin{array}{c}\text { Tempo } \\
(\text { min) }\end{array}$} & \multicolumn{2}{|c|}{$\begin{array}{c}\text { Cloro livre (mg Cl} \\
\left.\mathrm{L}^{-1}\right)\end{array}$} & \multicolumn{2}{|c|}{$\begin{array}{c}\text { Monocloramina }\left(\mathrm{mg} \mathrm{Cl}_{2}\right. \\
\left.\mathrm{L}^{-1}\right)\end{array}$} & \multicolumn{2}{|c|}{$\underset{\left.\mathrm{L}^{-1}\right)}{\operatorname{Dicloramina}\left(\mathrm{mg} \mathrm{Cl}_{2}\right.}$} \\
\hline & FH* & EC-FH & FH* & EC-FH & FH* & EC-FH \\
\hline 0 & na & na & na & na & na & na \\
\hline 60 & nd & 3 & 4 & 3,0 & 463 & nd \\
\hline 120 & nd & 2 & 7 & 5,0 & 585 & nd \\
\hline 180 & nd & nd & 10 & 1,0 & 899 & 5,0 \\
\hline 240 & nd & nd & 10 & nd & 930 & nd \\
\hline 300 & nd & nd & 11 & nd & 890 & nd \\
\hline 360 & nd & nd & 5 & nd & 865 & nd \\
\hline
\end{tabular}
destruídas.

Tabela 2. Evolução das concentrações de mono e di-cloraminas para FH* e EC-FH em função do tempo de irradiação.

Sem a remoção prévia do cloro pela EC as cloraminas formadas persistiram até 360 min de reação. Na FH a formação de dicloraminas foi máxima em 240 minutos (930 mg $\mathrm{Cl}_{2} \mathrm{~L}^{-1}$ ) e não houve nenhuma 
degradação destes compostos após este período.

A Resolução CONAMA $n^{\circ} 357$ (Brasil, 2005) estabelece os padrões de lançamento de efluentes industriais nos corpos hídricos brasileiros. Na Tabela 3 podem ser visualizados os valores de máxima eficiência obtidos para os parâmetros estudados nos menores tempos de tratamento para os processos EC e EC$\mathrm{FH}$, bem como os padrões de lançamento. Os valores de $\mathrm{pH}$ ficaram fora da faixa estabelecida pela legislação (5-9) tanto para o EC (60 min) que apresentou um $\mathrm{pH}$ de 11,8 quanto para EC-FH irradiando por 6 horas com pH final de 3,1. Em ambos os casos os valores de $\mathrm{pH}$ devem ser corrigidos antes do lançamento final. A legislação brasileira não estabelece limites de lançamento para os parâmetros DQO e COT. Os valores apresentados na Tabela 3 mostram que o efluente tratado pelo processo EC ainda apresenta uma carga orgânica residual $\left(\mathrm{DQO}=366 \mathrm{mg} \mathrm{O}_{2} \mathrm{~L}^{-1} \mathrm{e}\right.$ $\left.\mathrm{COT}=291 \mathrm{mg} \mathrm{C} \mathrm{L}^{-1}\right)$. Com o tratamento combinado houve elevada remoção da DQO $\left(3,5 \mathrm{mg} \mathrm{O}_{2} \mathrm{~L}^{-1}\right)$, porém não houve remoção significativa do COT $(215 \mathrm{mg} \mathrm{C}$ $\left.\mathrm{L}^{-1}\right)$.

$\mathrm{O}$ efluente tratado pelo EC apresentou $0,255 \mathrm{~g} \mathrm{Pt}-\mathrm{Co} \mathrm{L}^{-1}$ de cor o que não atendeu a legislação $\left(0,075 \mathrm{~g} \mathrm{Pt-Co} \mathrm{L}{ }^{-}\right.$ $\left.{ }^{1}\right)$, no entanto, para EC-FH o valor foi de 0,003, ficando bem abaixo do valor máximo estabelecido. Para os processos EC e EC-FH os valores de turbidez atenderam a legislação, uma vez que os resultados ficaram bem abaixo do limite máximo de lançamento (100 NTU), apresentando valores de 10,1 e 0,3 NTU, respectivamente.

Tabela 3. Valores dos parâmetros físico-químicos do efluente têxtil obtidos no tempo ótimo de tratamento para os processos EC, FH e EC-FH, comparados aos padrões de lançamentos de efluentes em corpos hídricos receptores (CONAMA, 357/2005).

\begin{tabular}{|c|c|c|c|c|}
\hline Parâmetro & ET & EC* & $\mathrm{EC}-\mathrm{FH} * * *$ & Padrões de lançamento \\
\hline $\mathrm{pH}$ & 12,5 & 11,8 & 3,1 & $5-9$ \\
\hline Nitrato $\mathrm{mg} \mathrm{NO}_{3}^{-} \mathrm{L}^{-1}$ & n.d. & 4,33 & 5,28 & n.e. \\
\hline Sulfato $\mathrm{mg} \mathrm{SO}_{4}^{2-} \mathrm{L}^{-1}$ & 2684 & 630 & 755 & 250 \\
\hline $\mathrm{N}$ amoniacal $\left(\mathrm{mg} \mathrm{NH}_{4}{ }^{+} \mathrm{L}^{-1}\right)$ & 1,33 & n.d. & 0,41 & $20 \mathrm{mg} \mathrm{N} \mathrm{L}^{-1}$ \\
\hline $\mathrm{N}$ orgânico $\left(\mathrm{mg} \mathrm{NH}_{4}^{+} \mathrm{L}^{-1}\right)$ & 3,24 & 2,70 & 0,72 & n.e. \\
\hline $\mathrm{DQO}\left(\mathrm{mg} \mathrm{O}_{2} \mathrm{~L}^{-1}\right)$ & 1636 & 366 & 3,5 & n.e. \\
\hline $\operatorname{COT}\left(\mathrm{mg} \mathrm{CL}^{-1}\right)$ & 636 & 291 & 215 & n.e. \\
\hline Turbidez (NTU) & 306 & 10,1 & 0,3 & 100 \\
\hline Cor $\left(\mathrm{g} \mathrm{Pt}^{-\mathrm{Co} \mathrm{L}^{-1}}\right)$ & 5,100 & 0,255 & 0,003 & 0,075 \\
\hline
\end{tabular}

*Eletrocoagulado por 60 minutos; ** Fotodegradado por 6 horas; *** Eletrocoagulado por 60 minutos e fotodegradado por 6 horas; n.a.: não analisado; n.d.: não detectado devido a presença de cor no efluente ou interferentes; n.e.: não especificado.

\subsection{TOXICIDADE DOS EFLUENTES TÊXTEIS TRATADOS POR EC, FH E EC-FH UTILIZANDO A ESPÉCIE ARTEMIA SALINA}

Os efluentes estudados apresentaram alta salinidade. A Artemia salina foi escolhida porque é pouco afetada por esta condição e desta forma a toxicidade estaria mais diretamente relacionada aos compostos formados durante os processos de tratamento, eliminando ou reduzindo a influência de íons comuns.

Com o número de artemias mortas, obtidos em cada ensaio, foi possível calcular os valores de $\mathrm{DL}_{50}$ para os processos de EC, FH e EC-FH que são mostrados na Tabela 4.

$\mathrm{O}$ tempo zero para $\mathrm{FH}$ e EC corresponde ao efluente têxtil não tratado diluído e não diluído, respectivamente. Para EC-FH corresponde ao efluente eletrocoagulado por 60 minutos. Nestes 
efluentes não foi possível calcular a $\mathrm{DL}_{50}$ devido à alta mortalidade das artemias em todas as diluições.

Tabela 4. Valores de $\mathrm{DL}_{50}$ obtidos com a espécie Artemia salina para os efluentes tratados por EC, $\mathrm{FH}^{*}$ e EC-FH.

\begin{tabular}{cccccc}
\hline \multicolumn{2}{c}{ EC } & \multicolumn{2}{c}{ FH* $^{*}$} & \multicolumn{2}{c}{ EC-FH } \\
\hline $\begin{array}{c}\text { Tempo } \\
(\mathrm{min})\end{array}$ & $\mathrm{LC}_{50}$ & $\begin{array}{c}\text { Tempo } \\
(\mathrm{min})\end{array}$ & $\mathrm{LC}_{50}$ & $\begin{array}{c}\text { Tempo } \\
(\min )\end{array}$ & $\mathrm{LC}_{50}$ \\
\hline 0 & $\mathrm{nc}$ & 0 & $\mathrm{nc}$ & 0 & $\mathrm{nc}$ \\
5 & $41 \pm 2$ & 60 & $\mathrm{nc}$ & 60 & $\mathrm{nc}$ \\
15 & $87 \pm 4$ & 120 & $35 \pm 2$ & 120 & $41 \pm 2$ \\
30 & $77 \pm 4$ & 180 & $65 \pm 3$ & 180 & $93 \pm 5$ \\
60 & $64 \pm 3$ & 240 & $46 \pm 2$ & 240 & $60 \pm 3$ \\
90 & $70 \pm 4$ & 300 & $68 \pm 3$ & 300 & $83 \pm 4$ \\
& & 360 & $75 \pm 4$ & 360 & $94 \pm 5$ \\
\hline
\end{tabular}

Após 5 minutos de eletrocoagulação, a redução da carga poluente promoveu uma redução benéfica na toxicidade para a espécie Artemia salina e atingiu um valor máximo de $87 \%$ para a $\mathrm{LD}_{50}$ em 15 minutos de reação.

Nos processos FH e EC-FH o comportamento da $\mathrm{DL}_{50}$ foi semelhante. Os valores da $\mathrm{DL}_{50}$ aumentaram até 180 min de irradiação, diminuíram em 240, voltaram a subir em $300 \mathrm{~min}$, sendo que os valores máximos da DL $_{50}$ foram 75 e $94 \%$ para $\mathrm{FH}$ e EC-FH, respectivamente, no tempo de $360 \mathrm{~min}$.

O aumento da toxicidade para ambos os tratamentos em $240 \mathrm{~min}$ de irradiação pode estar relacionada à presença de compostos intermediários formados durante os processos, que podem ter sido degradados posteriormente, uma vez que há novamente uma diminuição da toxicidade em 300 min de irradiação.

$\mathrm{O}$ teste com Artemia salina indicou uma menor toxicidade para o efluente tratado pelo processo combinado EC-FH, mas não foi observada uma correlação entre a toxicidade e a presença de mono e dicloraminas.

A toxicidade elevada no processo EC-FH nas duas primeiras horas de irradiação pode estar relacionada à presença de cloro livre, bem como pelo excesso de peróxido de hidrogênio.
O peróxido de hidrogênio possui reconhecida ação bactericida e algicida. A redução efetiva da concentração inicial de $\mathrm{H}_{2} \mathrm{O}_{2}$ é de suma importância, pois a presença de peróxido ao final das irradiações é caracterizada também como um tipo de resíduo que não é benéfico aos corpos receptores. No processo $\mathrm{FH}$ a concentração inicial de $\mathrm{H}_{2} \mathrm{O}_{2}$ foi de 0,05 e no EC-FH de $0,03 \mathrm{~mol} \mathrm{~L}^{-1}$.

As concentrações residuais de peróxido de hidrogênio após os tempos de irradiação das amostras tratadas pelos processos FH e EC-FH são mostradas na Figura 4.

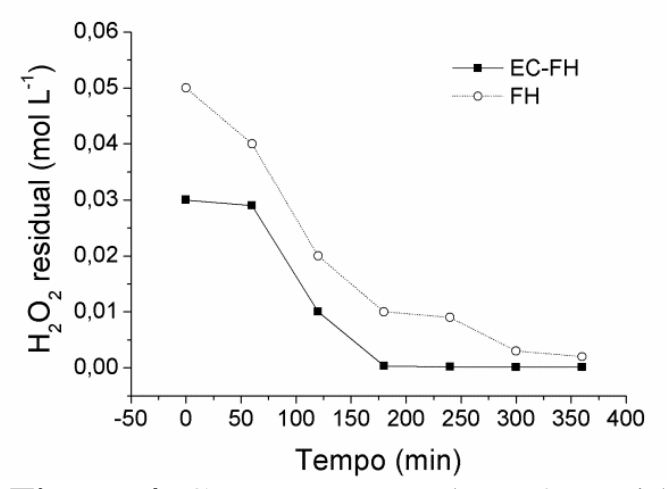

Figura 4. Concentrações de $\mathrm{H}_{2} \mathrm{O}_{2}$ residual em função do tempo de irradiação para os processos FH e EC-FH operando nas condições ótimas dos POR.

O consumo de peróxido foi superior a 99,0\% após 180 min de irradiação no processo EC-FH e alcançando 99,7\% após 300 min. No processo FH o consumo foi menos acentuado, sendo de 80,0\% em 180 min e 96,0 em 360 min. O aumento da toxicidade apresentado no tempo de 240 min, para os processos $\mathrm{FH}$ e EC-FH, não tem relação direta com as concentrações de peróxido residual e pode estar relacionado com a presença de outros compostos intermediários formados no processo de tratamento do efluente têxtil.

\section{CONCLUSÕES}

$\mathrm{O}$ processo EC sozinho não foi eficiente na remoção da cor do efluente, não atendendo os padrões de descarte impostos pela legislação brasileira vigente. 
A eficiência foi maior quando os processos $\mathrm{EC}$ e $\mathrm{FH}$ foram executados sequencialmente. $\mathrm{O}$ pré-tratamento pelo processo EC favoreceu a remoção de $60,3 \%$ do cloro total contido no efluente, reduzindo a formação de cloraminas durante a FH no tratamento combinado. As mono e dicloraminas formadas foram destruídas no processo EC-FH após 180 min de reação. A presença de peróxido de hidrogênio residual contribuiu para a elevada toxicidade, para a espécie Artemia salina, nas duas primeiras horas de irradiação. A toxicidade foi menor no tratamento EC-FH. De maneira geral o processo EC-FH foi superior na remoção da carga orgânica, apresentando-se como uma alternativa eficaz para o tratamento de efluentes têxteis clorados.

\section{AGRADECIMENTOS}

À CAPES pelo auxílio financeiro.

\section{BIBLIOGRAFIA}

APHA, AWWA, WPCF. 1998. Standard Methods for the Examination of Water and Wastewater, $20^{\text {st }}$ ed., American Public Health Association, Washington, DC.

APHA, AWWA, WPCF. 2005. Standard Methods for the Examination of Water and Wastewater, $21^{\text {st }}$ ed., American Public Health Association, Washington, DC.

AZBAR, N., YONAR, T. \& KESTIOGLU, K. 2004. Comparison of various advanced oxidation processes and chemical treatment methods for COD and color removal from a polyester and acetate fiber dyeing effluent. Chemosphere, 55, 35-43.

BARRAGÁN, B. E., COSTA, C. \& MÁRQUEZ, M. C. 2007. Biodegradation of azo dyes by bacteria inoculated on solid media. Dyes and Pigments, 75, 73-81.

BRASIL. Ministério do Meio Ambiente. Conselho Nacional do Meio Ambiente (CONAMA). 2005. Resolução $n^{\circ} 357$, de
17 de Março de 2005. Dispõe sobre a classificação e diretrizes ambientais para o enquadramento dos corpos de água superficiais, bem como estabelece as condições e padrões de lançamento de efluentes.

CHAKRABARTI, S. \& DUTTA, B. K. 2004. Photocatalytic degradation of model textile dyes in wastewater using $\mathrm{ZnO}$ as semiconductor catalyst. Journal of Hazardous Materials, 112, 269-278.

CHEN, G. 2004. Electrochemical technologies in wastewater treatment. Separation and Purification Technology, $38,11-41$.

DANESHVAR, N., OLADEGARAGOZE, A. \& DJAFARZADEH, N. 2006. Decolorization of basic dye solutions by electrocoagulation: An investigation of the effect of operational parameters. Journal of Hazardous Materials, 129, 116-122.

DENYER, P., SHU, L. \& JEGATHEESAN, V. 2007. Evidence of changes in membrane pore characteristics due to filtration of dye bath liquors. Desalination, 204, 296-306.

ECONOMIDES, D. G., VLYSSIDES, A. G., SIMONETIS, S. I. \& PHILIPPAKOPOULOU, T. L. 1998. Reuse of effluent from a wastepaper washdeinking process. Environmental Pollution, 103, 229-237.

EL-SHEEKH, M. M., GHARIEB, M. M. \& ABOU-EL-SOUOD, G. W. 2009. Biodegradation of dyes by some green algae and cyanobacteria. International Biodeterioration \& Biodegradation, 3, 699-704.

FIORENTIN, L. D., TRIGUEROS, D. E. G., MODENES, A. N., ESPINOZAQUIÑNONES, F. R., PEREIRA, N. C., BARROS, S. T. D. \& SANTOS, O. A. A. 2010. Biosorption of reactive blue 5G dye onto drying orange bagasse in batch 
system: Kinetic and equilibrium modeling. Chemical Engineering Journal, 163, 6877.

GARCIA, J. C., BOROSKI, M., OLIVEIRA, J. L., SILVA, A. E. C. \& NOZAKI, J. 2006. Solar and ultraviolet photodegradation of four textile dyes. In Trends in Solar Energy Research. New York: Nova Publishers.

GARCIA, J. C., OLIVEIRA, J. L., SILVA, A. E. C., OLIVEIRA, C. C., NOZAKI, J. \& SOUZA, N. E. 2007. Comparative study of the degradation of real textile effluents by photocatalytic reactions involving $\mathrm{UV} / \mathrm{TiO}_{2} / \mathrm{H}_{2} \mathrm{O}_{2}$ and $\mathrm{UV} / \mathrm{Fe}^{2+} / \mathrm{H}_{2} \mathrm{O}_{2}$ systems. Journal of Hazardous Materials, 147, 105-110.

GARCIA, J. C., SIMIONATO, J. I., SILVA, A. E. C., NOZAKI, J.\& SOUZA, N. E. 2009. Solar photocatalytic degradation of real textile effluents by associated titanium dioxide and hydrogen peroxide. Solar Energy, 83, 316-322.

GOGATE, P. R. \& PANDIT, A. B. 2004. A review of imperative technologies for wastewater treatment I: oxidation technologies at ambient conditions. Advances in Environmental Research, 8, 501-551.

GOLDER, A. K., HRIDATA, N., SAMANTA, A. N. \& RAY, S. 2005. Electrocoagulation of methylene blue and eosin yellowish using mild steel electrodes. Journal of Hazardous Materials, 127, 134140.

HAMILTON, M. A., RUSSO, R. C. \& THURSTON, R. V. 1997. Trimmed Spearman Karber statistical method for estimating median lethal concentration in toxicity bioassays. Environmental Science Technology, 11, 714-719.

KARRER, N. J., RYHINER, G. \& HINZLE, E. 1997. Applicabity test for combined biological-chemical treatment of wastewaters containing biorefractory compounds. Water Research, 31, 10131020 .

KIM, T. H., PARK, C., SHIN, E. B. \& KIM, S. 2002. Decolorization of disperse and reactive dyes by continuous electrocoagulation process. Desalination, 150, 165-175.

$\begin{array}{lll}\text { KRITIKOS, D. } & \text { E., } \\ \text { XEKOUKOULOTAKIS, } & \text { N. } & \text { P., }\end{array}$ PSILLAKIS, E. \& MANTZAVINOS, D. 2007. Photocatalytic degradation of Reactive Black 5 in aqueous solutions: effect of operatin conditions and coupling with ultrasound irradiation. Water Research, 41, 2236-2246.

KURBUS, T., MARECHAL, A. M. L. \& VONCINA, D. B. 2003. Comparison of $\mathrm{H}_{2} \mathrm{O}_{2} / \mathrm{UV}, \quad \mathrm{H}_{2} \mathrm{O}_{2} / \mathrm{O}_{3}$ and $\mathrm{H}_{2} \mathrm{O}_{2} / \mathrm{Fe}^{2+}$ processes for the decolorisation of vinysulphone reactive dyes. Dyes and Pigments, 58, 245-52.

MEYER, B. N., FERRIGNI, N. R., PUTNAM, J. E., JACOBSEN, L. B., NICHOLS, D. E. \& MCLAUGHLIN, J. L. 1982. Brine shrimp: a convenient general bioassay for active plant constituents. Journal of Medicinal Plant Research, 45, 31-34.

MOLLAH, M. Y. A., PATHAK, S. R., PATIL, P. K., VAYUVEGULA, M., AGRAWAL, T. S., GOMES, J. A. G., KESMEZ, M. \& COCKE, D. L. 2004. Treatment of orange II azo-dye by electrocoagulation (EC) technique in a continuous flow cell using sacrificial iron electrodes. Journal of Hazardous Materials, 109, 165-171.

OLIVEIRA, M. C., NOGUEIRA, R. F. P., GOMES-NETO, J. A., JARDIM, W. F. \& ROHWEDDER, J. J. R. 2001. Sistema de Injeção em Fluxo Espectrofotométrico Para Monitorar Peróxido de Hidrogênio em Processo de Fotodegradação por Reação Foto-Fenton. Química Nova, 24, 188-193. 
PALÁCIO, S. M., ESPINOZAQUIÑONES, F. R., MÓDENES, A. N., OLIVEIRA, C. C., BORBA, F. H. \& SILVA Jr, F. G. 2009. Toxicity assessment from electro-coagulation treated-textile dye wastewaters by bioassays. Journal of Hazardous Materials, 172, 330-337.

PALÁCIO, S. M., NOGUEIRA, D. A., MANENTI, D. R., MÓDENES, A. N., ESPINOZA-QUIÑONES, F. R. \& BORBA, F. H. 2012. Estudo da toxicidade de efluente têxtil tratado por foto-fenton artificial utilizando as espécies lactuca sativa e artemia salina. Engevista, 14, 127134.

PALÁCIO, S. M., PASTORIZA, M. C., MANENTI, D. R., BORBA, F. H., ESPINOZA-QUIÑONES, F. R. \& MÓDENES, A. N. 2013. Tratamento de efluente de cromagem por eletrocoagulação com eletrodo de aço inoxidável e eletrodo misto de aço inoxidável e alumínio. Engevista, 15, 248254.

PALIN, A. T. A. 1950. Study on the the chloro-derivatives of ammonia and related compounds, with special reference to their formation in the chlorination of natural and polluted waters. Water and Water Engineering, 54, 151-159 (October), 189200 (November), 248-256 (December).

PAPIC, S., VUJEVIC, D., KOPRIVANAC, N. \& SINKO, D. 2009. Decolourization and mineralization of commercial reactive dyes" by using homogeneous and heterogeneous Fenton and UV/Fenton processes. Journal of Hazardous Materials, 164, 1137-1145.

QAMAR, M., SAQUIB, M. \& MUNEER, M. 2005. Photocatalytic degradation of two selected dye derivatives, chromotrope 2B and amido black 10B, in aqueous suspensions of titanium dioxide. Dyes and Pigments, 65, 1-9.
RODRIGUES, A. C., BOROSKI, M., GARCIA, J. C., SHIMADA, N. S., NOZAKI, J. \& HIOKA, N. 2008. Treatment of paper pulp and paper mill wastewater by coagulation-flocculation followed by heterogeneous photocatalysis. Journal of Photochemistry and Photobiology A: Chemistry,194, 1-10.

SCOTT, K. 1995. Electrochemical Process for Clean Technology, The Royal Society of Chemistry, UK.

SHAKIR, K., ELKAFRAWY, A. F., GHONEIMY, H. F., BEHEIR, S. G. E. \& REFAAT, M. 2010. Removal of rhodamine B (a basic dye) and thoron (an acidic dye) from dilute aqueous solutions and wastewater simulants by ion flotation. Water Research, 44, 1449 - 1461.

SHI, B., LI, G., WANG, D., FENG, C. \& TANG, H. 2007. Removal of direct dyes by coagulation: The performance of preformed polymeric aluminum species. Journal of Hazardous Materials, 143, 567574.

SOUZA, R. R., BRESOLIN, I. T. L., BIONI, T. L., GIMENES, M. L. \& DIAS FILHO, B. P. 2004. The performance of a three-phase fluidized bed reactor in treatment of wastewater with high organic load. Brazilian Journal Chemical Engeneering, 21, 219-227.

TURABIK. M. 2008. Adsorption of basic dyes from single and binary component systems onto bentonite: Simultaneous analysis of Basic Red 46 and Basic Yellow 28 by first order derivative spectrophotometric analysis method. Journal of Hazardous Materials, 158, 5264.

USEPA (U.S. ENVIRONMENTAL PROTECTION AGENCY), EPA Drinking Water Criteria Document for Chloramines, Health and Ecological Criteria Division, Office of Science and Technology, Office of Water, 1994. Disponível em: 
http://www.epa.gov/ncea/water/chroramine /dwchoramine.pdf. Acesso em: 2012.

VILAR, V. J. P., CAPELO, S. M. S., SILVA, T. F. C. V. \& BOAVENTURA, R. A. R. 2011. Solar photo-Fenton as a preoxidation step for biological treatment of landfill leachate in a pilot plant with CPCs. Catalysis Today, 161, 228-234.

WHITE, G., 1999. Handbook of chlorination and alternative disinfectants. Van Nostrand Company. New York, 4 th. ed.

WU, C., CHANG, C. \& KUO, C. 2008. Decolorization of Procion Red MX-5B in electrocoagulation (EC), $\mathrm{UV} / \mathrm{TiO}_{2}$ and ozone-related systems. Dyes and Pigments, 76, 187-194.
SANTOS, S. M. \& GOUVEIA, N. 2011. Presença de trialometanos na água e efeitos adversos na gravidez. Revista Brasileira de Epidemiologia, 14, 1006-1019.

ZHANG, S. P. \& RUSLING, J. F. 1995. Dechlorination of polychlorinatedbiphenyls on soils and clay by electrolysis in a bicontinuous microemulsion. Environmental Science \& Technology, 29, 1195-1199.

POLCARO, A. M., PALMAS, S., RENOLDI, F. \& MASCIA, M. 1999. On the performance of $\mathrm{Ti} / \mathrm{SnO}_{2}$ and $\mathrm{Ti} / \mathrm{PbO}_{2}$ anodesin electrochemical degradation of 2chlorophenolfor wastewater treatment. Journal of Applied Eletrochemistry, 29, 147-151. 